Acta Cryst. (2002). A58 (Supplement), C151

CRYSTAL ENGINEERING OF THE GROWTH INTERFACE ${ }_{\text {N. Blagden }}^{1}$ R.J. Davey ${ }^{2}$ M. Song ${ }^{2}$

${ }^{1}$ University of Bradford School of Pharmacy Richmond Building BRADFORD WEST YORKSHIRE BD7 1DP UK ${ }^{2}$ Dept Chemical Engineering, UMIST Manchester M60 1QD

During the course of investigating the stabilisation of the meta-stable form of benzamide using additives it was observed that doping the aqueous supersaturated solution of benzamide with 2-amino-acetophenone (AAP) gave rise to an unusual growth behaviour which generates networks of crystals. The additive AAP appears to act as both an inhibitor and promoter of crystal nucleation and growth for benzamide. The resulting twinning and directed epitaxy resulted in a tiling of the growing benzamide crystals. A combined experimental and computational approach has been undertaken to examine this growth behaviour.

The evidence of date indicates that the crystal tiling mechanism appears to relate to a switch of growth direction arising from variations in the growth kinetics when the additive is present. The spatial arrangement of crystals indicates that the additive acts a molecular template to selectively direct the nucleation of one crystal onto another, in this role the additive acts like a molecular glue. In this role the additive exemplifies the subtleties that can be encountered when attempting to engineer the crystallisation, twinning and expitaxy of a molecular solid.

It is envisaged that this novel phenomenon could be exploited in drug delivery and agglomeration processing during crystallisation.

Keywords: ENGINEERING CRYSTAL INTERFACE

Acta Cryst. (2002). A58 (Supplement), C151

CRYSTAL ENGINEERING USING THE ANALOGY OF $X=O H$ AND

$\mathrm{NH}_{2}$ GROUPS IN 2-X-CYCLOALKANE-1-CARBOXYLIC ACIDS

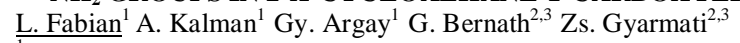

${ }^{1}$ Chemical Research Center, Hungarian Academy of Sciences Institute of Chemistry P.O. Box 17. BUDAPEST H-1525 HUNGARY ${ }^{2}$ Research Group for Heterocyclic Chemistry, Hungarian Academy of Sciences and University of Szeged ${ }^{3}$ Institute of Pharmaceutical Chemistry, University of Szeged

During a systematic investigation of the crystal structures of 2-OHcycloalkane-1-carboxylic acids it was found that they tend to form ladder-like hydrogen bond patterns [1]. The ladders are generated by hydrogen-bonded dimers. The dimers can be connected either parallel with or perpendicular to their principal axes [2]. Two patterns were identified that contain topologically identical arrays of inversion related dimers and differ only in the parallel vs. perpendicular direction of the attachment of the dimers.

The similar arrangement of the molecules in the two patterns suggested that a superposition of the two ladder patterns may be obtained by introducing new hydrogen bonds into the structure. Therefore, crystal structures of the homologous series of 2- $\mathrm{NH}_{2}$-cycloalkane-1-carboxylic acids with 6-8 membered rings were determined.

The three structures are isostructural, demonstrating the stability of their common hydrogen bond network. Although the compounds crystallize in zwitterionic form, this network confirmed our expectations. It is constructed of the two ladder patterns and the superposition of the perpendicular ladders leads to the formation of a two-dimensional hydrogen bond grid.

References

[1] A. Kalman, Gy. Argay, L. Fabian, G. Bernath, F. Fulop, Acta Cryst. (2001) B57, 539-550.

[2] A. Kalman, L. Fabian, Gy. Argay, G. Bernath, Zs. Gyarmati, Acta Cryst. (2002) B58, in the press.

Keywords: CRYSTAL ENGINEERING HYDROGEN BONDING ISOSTRUCTURALITY
Acta Cryst. (2002). A58 (Supplement), C151

\section{INTERACTION BETWEEN S IN S=C DOUBLE BONDS AND IODINE USING THE CSD}

M. Bolte $^{1}$ C. Naether ${ }^{2}$

${ }^{1}$ University of Frankfurt Organic Chemistry Marie-Curie-Strasse 11 FRANKFURT 60439 GERMANY ${ }^{2}$ University of Kiel

The preparation of new compounds with specific properties is one of the most important goals in modern chemistry. Since the crystal structure of a compound is directly linked to its properties it is desirable to control the crystallisation process. This strategy is described by the term 'Crystal engineering'. It stands for the design of special crystal structures with certain properties. Since the arrangement of the building blocks in a crystal depends on their mutual interactions, it is indispensable for a rational design of crystal structures to understand and use the interactions between these building blocks. One method to analyse these interactions is the systematic investigation of crystal structures with the aid of databases. We have concentrated on the investigation of interactions between iodine and $\mathrm{S}$ atoms in $\mathrm{S}=\mathrm{C}$ double bonds. The point at issue was whether the I atoms are distributed randomly around the $\mathrm{S}$ atoms or if there is any preferred orientation. We have found approximately 200 entries in the CSD which can be divided into two main groups. For an S...I distance between 2.5 and 3.2 Angstrom there is a distinct preferred orientation of the I atoms in direction of the lone pairs of the $\mathrm{S}$ atoms. Whereas at longer distances (3.5 to 5.0 Angstrom) the distribution of the I atoms is completely random. Furthermore, at short S...I distances the S...I-I angle is more or less 180 degrees. As a result of that, these interactions are of the donor-acceptor type. Additionally, there is a pronounced correlation between the intermolecular S...I and the intramolecular I-I distances. These data can be employed to derive a model for the electrophilic addition of iodine to thioketones resulting in the formation of iodonium cations and iodine anions.

\section{Keywords: DATA BASES CRYSTAL ENGINEERING STRUCTURE ACTIVITY RELATIONSHIP}

\section{Acta Cryst. (2002). A58 (Supplement), C151}

CRYSTAL AND MOLECULAR STRUCTURE OF DIETHYL TETRAHYDRO-1, 1-DIOXO-3, 5-DIPHENYL-1, 4-THIAZINE-2, 6DICARBOXYLATE

K.A.Nirmala KUSUMA ${ }^{1}$ Vasu $^{2}$ C. Choodamani ${ }^{3}$ R. Banurekha ${ }^{4}$ Bhavani ${ }^{4}$ ${ }^{1}$ Bangalore University Physics Jnanabharathi BANGALORE KARNATAKA 560056 INDIA

${ }^{2}$ Dept. of Physics, Vivekananda Degree College Malleshwaram, Bangalore560055. ${ }^{3}$ Dept. of Physics,Government Science College, Bangalore 560001. ${ }^{4}$ Dept. of chemistry, Annamalai University, Annamalainagar, Chidambaram.

The aim of science is understanding of observed phenomena as a consequence of laws of nature. Insights are gained into fundamental ordering principles that govern the great variety of observed events. In this respect X-ray crystallography has a special position in the scientific field. X-ray methods give the stereochemistry of the molecule which is not easy to characterize unambiguously compared to any other physical method like UV, NMR,IR etc, that is, some aspects of the structure of the title compound could not be interpreted with confidence by other methods. So the structural studies were taken up by $\mathrm{X}$-ray methods.

The title compound $\mathrm{C}_{22} \mathrm{H}_{25} \mathrm{NO}_{6} \mathrm{~S}$ is a intermediate derivative of Tetrahydro-1, 4-thiazine-1, 1-dioxide, which is used as sedatives, tranquillizers, antiepileptics, antitubercular,antitumour, bacteriocidal and paraciticidal agents. Tetrahydro-1, 4-thiazine-3,5-dicarboxylic acid has been detected in the bovine brain and human urine. The compound was crystallized from chloroform by slow evaporation method at room temperature. The crystals were colorless blocks, in the space group Triclinic with lattice parameters a $=10.217(1) \AA$, b $=10.713(2) \AA, c=11.397(1) \AA, \alpha=98.14(1)^{\circ}, \beta=109.38(1)^{\circ}, \gamma=107.25(1)^{\circ}$ with number of atoms $Z=2, \rho=1.323 \mathrm{mg} / \mathrm{m}^{3} \mathrm{~V}=1083.2(3) \AA^{3}$. The structure was determined by direct methods and refined to a final $\mathrm{R}$-value $=0.054$. There is disorder in one of the ethoxy group of the molecule, which could not be detected by any other method. The interesting results of the structure and conformation will be discussed in the presentation.

Keywords: SEDATIVES, THIAZINE, DISORDER 